\title{
Clinical Signs in Surgery: Are They Still Relevant?
}

\author{
Chintamani $^{1}$
}

Received: 13 December 2015 / Accepted: 13 December 2015 /Published online: 5 January 2016

(C) Association of Surgeons of India 2015

Teaching surgery is a great opportunity to learn and rediscover your own self through the eyes of your students, the taught thus becomes teacher's ultimate evaluator. Skilled clinical examination remains an important part of the management of any disease. While teaching and examining students across the country, one has observed that some traditional clinical tests of the beaten path that have long lost their relevance and utility continue to be part of the examination drill. A large number of these have a very poor sensitivity and specificity and are often cruel to the patients. We however continue to persist with them regardless. Whether there is something wrong with the tests or the teachers (or the taught) would need to be addressed. Like most clinical tests, the sensitivity of clinical signs is also expected to drop with time especially when more sensitive tools are universally available. It would therefore be mandatory to revisit these clinical tests in the light of present day and age, although it may amount to stumbling on the shoulders of giants.

To explain this point further, following are some amongst the many clinical signs that the author felt should be revisited.

1. Knee-elbow test: It was a very useful test in times with not much understanding of knee anatomy and its functionality. Current knowledge of the same makes this test noncompliant to the basic tenet of "primum non nocere". The unparalleled and universal availability of basic imaging

"For most diagnoses all that is needed is an ounce of knowledge, an ounce of intelligence, and a pound of thoroughness." Anonymous

Chintamani

drchintamani7@gmail.com

1 Vardhman Mahavir Medical College, Safdarjang Hospital, New Delhi, India modalities makes this test redundant in rendering any clinical insight. The test has lost its significance and utility in the age of ultrasound and other inexpensive non-invasive ways. The patient is expected to get into a very uncomfortable position and the examinee is often searching for the space between examination couch and the abdominal wall to locate a lump that is supposed to fall forward if it is intra-peritoneal. Indeed, it is a great effort to achieve very little. A "shift test" that many authors use quite frequently in their clinical practice for this purpose is a modification that is easily reproducible and can be as effective with minimal or no discomfort to the patient. The patient has simply to be moved into a lateral position after the lump has been marked while the patient was supine, and if the lump moves, it is more likely to be intra-peritoneal while retroperitoneal lumps are fixed.

2. Puddle's sign for minimal ascites: With the patient in knee-elbow position, the examinee is expected to percuss in the little space between examination couch and patient's tummy to detect the miniscule amount of ascites. Often a poorly performed test, it is neither patient friendly nor yields any conclusive information.

3. Percussing on sternum for retrosternal extension of the thyroid gland: Percussion note is often dull on the bone even if it is cancellous, and examinees often modify their findings in the light of other clinical signs.

4. Finger invagination test for groin hernia: Taught for long as an essential part of groin examination, the test is not only patient unfriendly but also scientifically unnecessary. With the current understanding of groin hernia management, the size of the superficial ring, the direction of the hernia sac and the tone of the conjoint tendon are not relevant clinically. The author has witnessed patients collapsing due to vasovagal phenomenon with a slight deviation in reproducing the test. 
5. Rebound tenderness: Eliciting rebound tenderness is one of the most scientific clinical tests. While it points to the presence of peritoneal insult, it insults the patient comfort too. To cause pain while evaluating to achieve relief from pain deserves revision in our methodology. The objective of parieto-visceral marriage in adversity can easily be elicited simply by asking the patient to perform the Valsalva manoeuvre. Some authors also practice the "pinch-an-inch" test where a skin fold of the abdomen over suspected quadrant is grasped and pulled away from the peritoneum. The lifted skin fold is then released to recoil briskly, leading to increased pain when it strikes the inflamed peritoneum.

There is a long laundry list of these tests that would need to be updated and relooked in order for them to remain relevant. Current surgical training demands compulsory skills for surgeons in imaging modalities especially ultrasound and various endoscopies as an extension of clinical examination. Ultrasound examination is now considered an extension of clinical examination (surgeon's stethoscope). These modalities would easily settle most of the above-mentioned issues conclusively without potential discomfort to the patient. The ultrasound examination is both inexpensive and non-invasive and can be easily taught to all trainees and can be used even in the remotest of places.

While examining at a postgraduate examination centre, the author has been witness to a patient with obstructive jaundice collapsing during the "knee-elbow test". It is certainly not acceptable to persist with such clinical signs unless we update and modify them suitably so that they are more sensitive and patient friendly rather than letting them simply remain as part of an examiner's/examinee's checklist. There is a need to have rationalization of our clinical approach that needs to parallel the rapid technological evaluation.

While many clinically unproductive clinical signs are still persisted, simpler and more rewarding tests like digital rectal examination (DRE) are being forgotten both for the teacher and the taught. A simple DRE is a very sensitive digital bio-probe to evaluate local as well as locoregional pathologies, state of digestive tract and even neuromuscular status of the patient. Signs less relevant are enforced for the urge of fancy display while DRE is omitted for a variety of reasons. It allegedly takes too much time, is not friendly to our olfactory sensibilities and is not aesthetically pleasing. However, as was so often repeated by surgical teachers in the past "if you don't put your finger in to it, you have put your foot in to it", the test continues to be relevant. There is also a strong need to educate the trainers into putting it across to their trainees in a manner that they learn these clinical signs with patient in mind rather than just to pass an examination.

"When I was preparing for my most recent exams we were acutely aware that failure of finals was virtually synonymous with failure of the clinical examination components and consequently we practiced little else. Now, I would never want to deny that good and thorough clinical examination is anything other than a cornerstone of good medical practice; but bearing in mind that I have just begun work unable to dose warfarin, barely competent at bag mask ventilation, and struggling to remember the anaphylaxis dose of epinephrine (is it $0.5 \mathrm{ml}$ of $1: 1000$ or $1: 10000$ ?) I can't help but wonder whether learning to spot Kayser-Fleischer rings was time well spent. Still, maybe one day it will come up in a pub quiz..." (Will Muirhead BMJ 2010; 341:c5205). 